\title{
ADN ancien et évolution humaine : nouveaux outils, nouvelles perspectives
}

Caroline COSTEDOAT

Stéphane MAZIĖRES

Étonnamment, l'histoire de l'« ADN ancien » (noté parfois ADNa) est une histoire qui a commencé il y a juste 35 ans. C'est en effet, en 1984, au balbutiement de la biologie moléculaire, qu'une équipe californienne (Higuchi et al., 1984) parvient à cloner un fragment d'ADN d'un quagga, une sous-espèce de zèbre éteinte à la fin du XIX ${ }^{\mathrm{e}}$ siècle. Cette découverte revenait à dénicher alors l'impensable : des reliquats "vivants" (organiques) dans du non-vivant. Par cette ressuscitation in vitro de l'information génétique ancienne, la communauté scientifique mesurait alors le nouveau potentiel de cette découverte. S'ouvraient ainsi les débuts de la paléogénétique, à la croisée de deux champs disciplinaires : la paléontologie ${ }^{1}$ et la phylogénie moléculaire ${ }^{2}$. Les paléontologues sont confrontés aux données fossiles, souvent incomplètes et/ou mal conservées. L'extrapolation d'une histoire évolutive est donc à ce niveau complexe et conduit inexorablement à la naissance de plusieurs hypothèses, entre lesquelles il est souvent bien difficile de trancher. Quant à la phylogénie moléculaire, elle est jusqu'alors cantonnée à la comparaison d'espèces actuelles et les relations de parentés construites souffrent d'un manque de profondeur, de données ancestrales pour polariser les relations entre les différents groupes. Deux approches différentes donc, l'une comparative, l'autre analytique, sur des échelles de temps distinctes. L'enjeu de la paléogénétique était clair : concilier ces deux approches. Il devenait alors évident que nous pouvions franchir un nouveau cap et étudier des séquences humaines du passé, nous permettant ainsi d'aborder l'évolution humaine par un prisme différent. On allait pouvoir se pencher sur des questions d'évolution au niveau des gènes plutôt que de se fier uniquement à la variation phénotypique, et ce, grâce à la génétique et aux nouveaux outils moléculaires en émergence. Aujourd'hui, à partir d'ossements, de dents, de cheveux, de restes organiques ou encore de sédiments, il est possible de reconstituer de l'ADN ancien, même lorsqu'il a subi des fragmentations ou des modifications chimiques dues au temps 
ou à l'environnement. La révolution technologique que connaît la biologie moléculaire associée au progrès bioinformatique nous permettent de rentrer dans l'aire de la paléogénomique où des génomes dit " archaïques » sont révélés et livrent leurs secrets, nous éclairant sur l'histoire des communautés passées, nous permettant d'aborder des problématiques jusque-là non envisagées.

Dans les lignes qui suivent, nous aborderons les challenges techniques et méthodologiques qui ont conduit à l'émergence de cette nouvelle discipline. Nous passerons ensuite en revue les grandes questions abordées par l'étude de l'ADN ancien humain. Nous verrons comment certaines promesses ont pu être concrétisées, alors que d'autres n'ont pas encore trouvé de réponse. Enfin, nous esquisserons les perspectives en abordant certaines des questions encore ouvertes aujourd'hui.

\section{Des prouesses méthodologiques aux biais technologiques et vice-versa : naviguer dans un monde fragile}

L'histoire de la paléogénétique est jalonnée de résultats quelques peu critiquables qui n'ont pas manqué d'entourer cette discipline d'un scepticisme général. Mais c'est aussi un champ disciplinaire qui fait rêver et qui a très vite été médiatisé attirant ainsi le grand public et offrant des scénarios de science-fiction qui ont explosé sur grand écran (e.g. avec le film Jurassic Park). L'ADN ancien est souvent présent en petite quantité, il est fragile, très fragmenté et la contamination avec de l'ADN moderne est courante. Dans ce contexte, obtenir " le vrai » devient un vrai challenge contraint par les progrès méthodologiques et technologiques. Dans les cellules vivantes, il existe des mécanismes de réparation de l'ADN qui n'existent plus après la mort. A ce moment-là les dommages subis par l'ADN s'accumulent conduisant à des mutations, des coupures de brins d'ADN et la perte de certaines bases. Ces facteurs de dégradation (oxydation et hydrolyse) sont influencés par des variables environnementales ( $\mathrm{pH}$, températures...) qui accélèrent ou au contraire ralentissent la dégradation de l'ADN. Les glaces, les tourbières ou les déserts chauds sont des environnements favorables. Ces environnements peuvent, dans certains cas, garder en leur sein des restes anciens momifiés sur lesquels il est possible d'extraire de l'ADN à partir de tissus " mous ». Mais la momification peut également être artificielle, liée à l'action de l'Homme (pratiques funéraires, taxidermie). 
Les premières données d'ADN ancien sur la lignée humaine sont issues de tissus d'une momie égyptienne datant d'environ 2400 ans (Pääbo, 1985). Une véritable révolution qui a très vite suscité l'intérêt de toute la communauté scientifique mais également du grand public. Or, ces premières séquences d'ADN ancien humain se sont avérées être par la suite une contamination avec de l'ADN actuel liée notamment à l'utilisation du clonage moléculaire par transformation bactérienne ${ }^{3}$, technique nécessitant un matériel de départ de très bonne qualité. Nous sommes à cette époque aux balbutiements de la $P C R^{4}$ qui n'est pas encore utilisée en routine. Mais l'enthousiasme provoqué par ces études d'ADNa va très vite conduire les chercheurs à utiliser cette nouvelle méthode d'amplification (qui permet de détecter des quantités négligeables d'ADN) et qui deviendra l'outil de prédilection des paléogénéticiens. Cependant, très vite là encore, les premiers biais sont identifiés. En effet, si elle est utilisée sans précautions particulières, la PCR peut donner des résultats trompeurs, et dans le cas d'ADN très fragmenté l'amplification enzymatique peut produire des chimères. Ainsi, plus qu'une prouesse technologique c'est toute une démarche méthodologique qui a été mise en place pour l'étude d'ADNa où l'analyse de séquences d'ADN est en fait le dernier maillon. Une méthodologie de laboratoire précise sera mise en place pour l'ADNa et améliorée au fil du temps afin de limiter les contaminations a priori et a posteriori. Mais en parallèle de ces progrès " de cuisine ", il fallait pouvoir se détacher des tissus momifiés. En effet, ce matériel reste rare et est souvent géographiquement et temporellement circonscrit. Une étape très importante de la discipline a été de réussir des amplifications d'ADNa à partir de restes archéologiques "durs " (os, dents...) ouvrant des dimensions géographiques et temporelles beaucoup plus importantes.

La recherche sur l'ADN ancien est maintenant arrivée à maturité avec des procédures réplicables et rigoureuses. En 2010, c'est-à-dire une décennie après la caractérisation du génome humain de référence, la première séquence du génome humain ancien a été publiée. Avec le débit massif des plateformes dites de séquençage nouvelle génération $\left(\mathrm{NGS}^{5}\right.$ ) les génomes s'enchaînent (figure 1).

Le premier génome humain ancien a été séquencé avec une couverture élevée (20 X5) en 2010 à partir d'un bulbe capillaire d'un Eskimo groenlandais vieux d'environ 4000 ans (Rasmussen et al., 2010). Jusqu'à cette époque, il était généralement admis qu'aucune séquence d'ADN fiable ne pouvait être extraite de restes humains anatomiquement modernes en raison de problèmes de contamination. Pourtant, aujourd'hui, plus de 500 génomes humains anciens ont été analysés y compris les individus du Paléolithique supérieur (exemple dans Fu et al., 2014), des Amérindiens préhistoriques et historiques (exemple dans Rasmussen et al., 2014), des chasseurs-cueilleurs du Mésolithiques 
Figure 1. Trente-cinq ans de recherche en ADNa : quelques étapes clefs dans l'étude de l'évolution humaine.

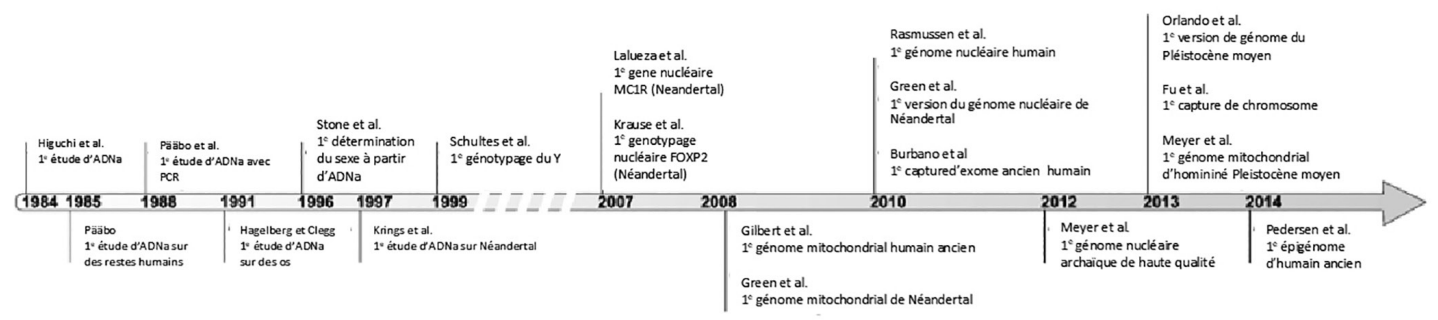

(exemple dans Lazaridis et al., 2014), des chasseur-cueilleurs et fermiers du Néolithiques (exemple dans Gamba et al., 2012), des Eurasiens de l'âge de bronze (exemple dans Cassidy et al., 2016), des échantillons historiques (exemple dans Schiffels et al., 2016).

En parallèle de ces génomes d'Hommes anatomiquement moderne, la première ébauche du génome Néandertal a été publiée en 2010, ouvrant la génomique à l'étude des homininés archaïques (Green et al., 2010). Peu de temps après, une nouvelle espèce, totalement inconnue des anthropologues, a été découverte sur la base de la signature génétique divergente à partir de l'os d'une phalange : " l'Homme de Denisova », du nom de la grotte où le petit fragment osseux a été excavé (Reich et al., 2010). Depuis lors, des génomes de haute qualité provenant à la fois de Néandertaliens et de dénisoviens ont été générés ainsi que des génomes encore provisoires d'autres individus néandertaliens.

Outre les défis méthodologiques et technologiques l'analyse de ces génomes nous renseigne sur les origines et la composition génétique de notre propre espèce, et produit des preuves d'un mélange ancien entre différentes lignées d'hominidés. Chaque nouveau génome ancien apporte sa pierre à l'édifice dans la compréhension de la diversité génétique humaine comme nous l'illustrons dans les paragraphes suivants.

\section{Peuplements holocènes : expansions et (dis)continuité génétique}

Alors que les grandes tendances de la diversité génétique actuelle en dehors de l'Afrique ont été formées par le goulot d'étranglement hors d'Afrique et la divergence qui s'est ensuivie, une grande partie de la structure d'échelle plus fine est le résultat de mouvements de population relativement récents. Dans de nombreux cas, l'ADN ancien permet de résoudre des questions sur 
les processus historiques qui façonnent la structure génétique des populations et qui ne pourraient pas être résolues par les seuls génomes actuels. Dans cette section, nous passons en revue les résultats récents obtenus dans différentes parties du monde et discutons de ce que l'ADN ancien nous a appris en général sur les processus qui ont formé la diversité génétique actuelle.

Les masses continentales Eurasiatique et Américaines rassemblent l'essentiel des études de paléogénétique, notamment parce qu'elles s'étendent au-delà des latitudes tempérées, offrant de meilleures conditions taphonomiques. Les grandes problématiques abordées concernent l'expansion des premiers agriculteurs en Méditerranée et dans le bassin du Danube, les expansions postglaciaires scandinaves, les mouvements steppiques de l'Âge du Bronze, l'introduction de l'agriculture au Japon et les mouvements des premiers amérindiens arctiques. Conjointement à la séquence spatio-temporelle de ces peuplements, les travaux permettent de faire le point sur le devenir des patrimoines génétiques de ces populations mésolithiques et néolithiques. Les données de l'ADN ancien de restes Aurignaciens de Russie (Kostenki 14, 37000 ans) d'Europe de l'Ouest (Goyet 116.1, 35000 ans) et du Néolithique (La Brana 1, 7000 ans) ont révélé qu'une lignée génétique masculine aujourd'hui disparue en Europe est observée à l'Est de l'Oural, signifiant pour l'Europe que le patrimoine génétique des populations pré-néolithiques de chasseurs-cueilleurs a disparu. De la même façon, les résultats de l'étude de restes néolithiques et de l'Age des Métaux, entre 5000 et 3000 ans avant notre ère, montrent une tendance similaire à l'arrivée du Néolithique en Europe, avec à nouveau un remplacement démique et culturel des populations déjà établies. La discontinuité génétique à la transition de périodes culturelles majeures n'est pas une généralité. Pour les régions aux voies d'accès étroites, comme le continent américain, l'étude diachronique de son peuplement, notamment par l'exploration des systèmes de groupes sanguins, montre au contraire une continuité des patrimoines génétiques entre les périodes anciennes (10000 ans avant notre ère) et l'actuel (exemple sur le système $\mathrm{ABO}$ ). Récemment, l'Anthropologie est parvenue à descendre sous le $40^{\circ}$ parallèle et à explorer pour la première fois les patrimoines génétiques anciens d'Afrique (Sud et Est uniquement). Ces régions sont les destinations terminales des grands mouvements linguistiques et d'un mode de vie pastoral et agricultural survenus à l'aube de notre ère, dont le plus connu est l'expansion Bantu.

Les grandes lignes de l'histoire humaine ne sont pas très différentes de ce que Cavalli-Sforza a pu deviner sur la base d'autres marqueurs (tels que les groupes sanguins, les protéines et enzymes du sang). Cependant, des modèles de plus en plus détaillés de remplacement, de migration et de métissage, qui étaient auparavant invisibles pour les chercheurs, émergent dans les régions où l'ADN est disponible en 
abondance. Nous savons maintenant que les populations actuelles sont issues d'une histoire complexe de mélanges et de mouvements de populations. Bien que la continuité génétique locale sur de longues périodes ait été documentée dans quelques cas, ceux-ci sont exceptionnels. La règle générale est que les ancêtres des populations d'aujourd'hui ont vécu ailleurs ou ont été totalement remplacés (Skoglund \& Mathieson, 2018).

\section{Les gènes, comme les corps, se sont entrelacés (variation fonctionnelle, phénotypes et sélection)}

L'étude directe de l'ADN peut porter sur différents types de marqueurs : les marqueurs nucléaires diploïdes biparentaux et les marqueurs haploïdes uni-parentaux. Les premiers sont portés par les 22 paires d'autosomes et la paire de chromosomes $X$ chez la femme, ils représentent $50 \%$ de la contribution paternelle et $50 \%$ de la contribution maternelle. Les seconds sont portés par l'ADN des mitochondries - transmis que par la mère-et le chromosome $Y$, typiquement masculin. Ils échappent à tout processus de recombinaison et les seules variations possibles sont les mutations qui s'accumulent sur le même segment ADN sans jamais être séparées d'une génération à l'autre. Ce maintien groupé des mutations qui s'accumulent de génération en génération permet alors une construction phylogénétique de ces différents marqueurs. L'ADN mitochondrial a la particularité d'être présent en milliers d'exemplaires par cellule, ce qui en fait un marqueur de choix pour l'étude de l'ADN des restes anciens. Le premier défi de l'étude de
l'ADN ancien consistait donc à parvenir à amplifier l'ADN nucléaire autosomal, plus rare par cellule mais détenant l'essentiel du patrimoine génétique. Dans l'histoire de l'étude de l'ADN ancien, ce n'est qu'à partir de 2010 que les travaux génétiques sur l'ancien basculèrent pleinement de l'ADN mitochondrial à l'ADN autosomal. C'est l'ensemble des régions fonctionnelles qui devient désormais accessible et ainsi la possibilité de savoir à quoi ressemblaient nos ancêtres et l'histoire des gènes qui nous constituent aujourd'hui. Et s'il n'y a pas d'os ? Pas de problème : l'ADN laissé dans le sédiment des grottes peut révéler une ancienne occupation humaine (Slon et al., 2017). Bien qu'il ait déjà été démontré que l'Homme de Neandertal a contribué à l'ADN des humains modernes, l'étude diachronique des populations Néandertalienne et Dénisovienne a révélé une diversité génétique élevée au sein de ces populations archaïques, avec un changement majeur de populations chez ceux du Caucase et d'Europe par rapport aux populations de Sibérie, ainsi 
qu'un flux de gènes unidirectionnel, de Neandertal à l'Homme moderne et pas l'inverse. Cette contribution des espèces archaïques au patrimoine génétique humain actuel concerne notamment des régions fonctionnelles, et celles-ci diffèrent d'une population à l'autre.

Il semble que le métissage Neandertal/Homme moderne ait été bénéfique pour conquérir les territoires en dehors de l'Afrique et s'adapter aux environnements peu familiers de l'Eurasie. Un exemple de cela est une région $\mathrm{ADN}$ partagée avec le génome de l'Homme de Denisova, à haute fréquence chez les Tibétains d'aujourd'hui, et qui semble être une adaptation à la vie à haute altitude, mais le rôle de cette région ADN dans la physiologie de Denisova n'est pas clair. Des signes de mélange archaïque adaptatif ont également été trouvés dans les populations Européennes, si bien que l'ADN ancien a contribué directement à notre compréhension des origines des variations phénotypiques suivantes : système immunitaire, métabolisme (persistance de la lactase), structure et pigmentation de la peau, des yeux et des cheveux. On ignore cependant si l'ascendance archaïque contribue aux différences systématiques entre les populations actuelles. Les proportions d'ascendance néandertalienne ne varient que d'une petite quantité au sein de l'Eurasie $(\sim 2-2,5 \%)$, et cette ascendance provient probablement de la même population néandertalienne, mais les plus grandes différences d'ascendance archaïque entre les populations africaines, eurasiennes et océaniennes d'aujourd'hui pourraient expliquer une plus grande proportion de différences phénotypiques. Nous avertissons toutefois que ces différences dans l'ascendance archaïque sont confondues avec la différenciation génétique non archaïque, de sorte qu'il sera difficile de concevoir des études pour résoudre cette question.

De la même manière que l'ADN ancien a significativement contribué à expliquer l'origine de la variabilité en termes de processus historiques, l'ADN ancien devrait également nous permettre d'éclairer la base génétique de la diversité phénotypique humaine. En particulier, l'ADN ancien devrait être précieux pour quantifier la contribution de la sélection naturelle à la variation phénotypique, puisqu'il fournit des données directes sur l'évolution. La sélection naturelle historique laisse des modèles détectables dans les génomes actuels, mais ces signatures peuvent être difficiles à interpréter et peuvent être imitées par des processus neutres. L'ADN ancien nous permet de détecter directement les changements rapides dans les fréquences des allèles au fil du temps - les résultats immédiats de la sélection. Enfin, l'étude comparée des génomes actuels, Neandertalien et Denisovien, révèle qu'il aurait existé une sous-espèce de sapiens ancestrale à ces trois populations, à partir de laquelle Denisova se serait différencié il y a un peu plus de 900000 ans et dont l'héritage est estimé entre 0,5 et $8 \%$ de l'ADN de l'Homme de Denisova (Prufer et al., 2014). Ce résultat est frappant car c'est 
la première fois que la génétique met au jour l'existence d'un « corps » avant l'archéologie ; habituellement la pipette du généticien intervient après la truelle de l'archéologue.

\section{Perspectives}

Méthodologiques : les environnements favorables à la préservation de l'ADN (tourbières, permafrosts...) regroupent des conditions très exceptionnelles. Cependant, la plupart des restes archéologiques et paléontologiques sont issus de gisements qui ne présentent pas ces caractéristiques remarquables. L'enjeu actuel est donc d'essayer de cerner les relations " ADN-environnement » afin de mieux appréhender les processus de dégradation en fonction du milieu d'échantillonnage. C'est donc un véritable travail de taphonomie moléculaire qui est actuellement à engager.

Spatiale : descendre en latitude, explorer l'Afrique sub-saharienne ancienne. La découverte génomique est analogue à lâge de la découverte géographique débridée par les Européens des $\mathrm{XVI}^{\mathrm{e}}$ et XVII siècles. Presque tous les génomes nucléaires anciens offrent de nouvelles perspectives sur l'histoire humaine et ouvrent de nouveaux horizons de recherche anthropologiques. $\mathrm{Si}$ les régions tempérées et arctiques ont délivré beaucoup plus de séquences génétiques anciennes, en partie parce qu'elles sont plus favorables à la préservation des corps et qu'elles ont été plus intensément échantillonnées, l'extraction récente des génomes anciens des Monts Zagros (Iran, $35^{\circ} \mathrm{N}$ ), de la grotte de Mota (Éthiopie, ${ }^{1} 1^{\circ} \mathrm{N}$ ), d'Afrique $\mathrm{du}$ Sud et de $\mathrm{l}^{\prime}$ Est en général $\left(3-32^{\circ} \mathrm{S}\right)$ montre qu'il est possible d'outrepasser le $30^{\circ}$ parallèle et de pénétrer dans la frange inter-tropicale, zone de la plus grande diversité de biotope, biocénose, pathocénose et de variabilité génétique humaine, siège de l'origine de l'Homme anatomiquement moderne.

Temporelle : descendre le temps, de la préhistoire à l'histoire. L'ADN ancien de l'époque historique est encore relativement rare, en partie parce qu'il est plus difficile d'évaluer la contamination des individus d'aujourd'hui. Cependant, un nombre croissant d'études ont commencé à aborder ces périodes, y compris des études sur les changements dans l'ascendance en GrandeBretagne pendant les périodes romaine et anglo-saxonne, le peuplement des îles Canaries, la réduction dramatique de la population amérindienne qui a suivi les contacts européens, ou encore la localisation grâce à la génétique du lieu d'origine des esclaves africains dans les Caraïbes. Mais l'utilisation de l'outil 
génétique fait son apparition dans des contextes encore plus récents. Nous citerons l'identification de soldats disparus de la grande guerre, ou encore l'identification d'un élève pilote allemand de la seconde guerre mondiale (Costedoat et al., 2019).

L'analyse de ces données archéocontemporaines exigera que les généticiens s'engagent avec les historiens, comme ils l'ont déjà fait avec les anthropologues et les archéologues pour interpréter les données d'individus plus anciens. Enfin, alors que l'analyse de l'ADN ancien a été historiquement concentrée dans les laboratoires européens et nord-américains, l'expansion des études de l'ADN ancien à d'autres parties du monde - et à des périodes plus récentes - implique à la fois une opportunité et une obligation de développer plus largement les capacités d'analyse.

\section{Bibliographie}

Cassidy LM., Martiniano R., Murphy EM., Teasdale MD., Mallory J., Hartwell B. Bradley D.G. 2016, « Neolithic and Bronze Age migration to Ireland and establishment of the insular Atlantic genome ", dans PNAS, $n^{\circ}$ 113: 368-373.

Costedoat C., Adalian P., Bouzaid E., Martinet A., Vanrell L., Von Gartzen L., Castellano P., Signoli M., Tzortzis S., Stevanovitch A. 2019, "When a lost "Petit Prince" meets Antoine de Saint Exupéry: an Anthropological story", dans Forensic Science International, doi.org/10.1016/j.forsciint.2019.01.015.
Fu Q., Li H., Moorjani P., Jay F., Slepchenko S.M., Bondarev A.A., Johnson P.L., AximuPetri A., Prüfer K., de Filippo C., Meyer M., Zwyns N., Salazar-García D.C., Kuzmin Y.V,. Keates S.G., Kosintsev P.A., Razhev D.I., Richards M.P., Peristov N.V., Lachmann M., Douka K., Higham T.F., Slatkin M., Hublin J.J., Reich D., Kelso J., Viola T.B., Pääbo S. 2014, "Genome sequence of a 45,000-year-old modern human from western Siberia", dans Nature, n' 514 : 445-449. Gamba C., Fernandez E., Tirado M., Deguilloux M.F., Pemonge M.H., Utrilla P., Edo M., Molist M., Rasteiro R., Chikhi L., Arroyo-Pardo E. 2012, « Ancient DNA from an Early Neolithic Iberian population supports a pioneer colonization by first farmers », dans Molular Ecology, n² 21: 45-56.

Green R.E., Krause J., Briggs A.W., Maricic T., Stenzel U., Kircher M. 2010, Patterson N., Li H., Zhai W., Fritz M.H., Hansen N.F., Durand E.Y., Malaspinas A.S., Jensen J.D., Marques-Bonet T., Alkan C., Prüfer K., Meyer M., Burbano H.A., Good J.M., Schultz R., Aximu-Petri A., Butthof A., Höber B., Höffner B., Siegemund M., Weihmann A., Nusbaum C., Lander E.S., Russ C., Novod N., Affourtit J., Egholm M., Verna C., Rudan P., Brajkovic D., Kucan Ž., Gušic I., Doronichev V.B., Golovanova LV., Lalueza-Fox C., de la Rasilla M., Fortea J., Rosas A., Schmitz R.W., Johnson P.L.F., Eichler E.E., Falush D., Birney E., Mullikin J.C., Slatkin M., Nielsen R., Kelso J., Lachmann M., Reich D., Pääbo S. «A draft sequence of the Neandertal genome ", dans Science, $\mathrm{n}^{\circ} 328: 710-722$.

Higuchi R., Barbara B., Freiberger M., Ryder O., Wilson A.C. 1984, "DNA sequences from the quagga, an extinct member of 
the horse family ", dans Nature, $\mathrm{n}^{\circ} 312$ : 282-284.

Lazaridis I., Patterson N., Mittnik A., Renaud G., Mallick S., Kirsanow K., Sudmant P.H., Schraiber J.G., Castellano S., Lipson M., Berger B., Economou C., Bollongino R., Fu Q., Bos K.I., Nordenfelt S., Li H., de Filippo C., Prüfer K., Sawyer S., Posth C., Haak W., Hallgren F., Fornander E., Rohland N., Delsate D., Francken M., Guinet J.M., Wahl J., Ayodo G., Babiker H.A., Bailliet G., Balanovska E., Balanovsky O., Barrantes R., Bedoya G., Ben-Ami H., Bene J., Berrada F., Bravi C.M., Brisighelli F., Busby GB., Cali F., Churnosov M., Cole D.E., Corach D., Damba L., van Driem G., Dryomov S., Dugoujon J.M., Fedorova S.A., Gallego Romero I., Gubina M., Hammer M., Henn B.M., Hervig T., Hodoglugil U., Jha A.R., Karachanak-Yankova S., Khusainova R., Khusnutdinova E., Kittles R., Kivisild T., Klitz W., Kučinskas V., Kushniarevich A., Laredj L., Litvinov S., Loukidis T., Mahley R.W., Melegh B., Metspalu E., Molina J., Mountain J., Näkkäläjärvi K., Nesheva D., Nyambo T., Osipova L., Parik J., Platonov F., Posukh O., Romano V., Rothhammer F., Rudan I., Ruizbakiev R., Sahakyan H., Sajantila A., Salas A., Starikovskaya E.B., Tarekegn A., Toncheva D., Turdikulova S., Uktveryte I., Utevska O., Vasquez R., Villena M., Voevoda M., Winkler C.A., Yepiskoposyan L., Zalloua P., Zemunik T., Cooper A., Capelli C., Thomas M.G., Ruiz-Linares A., Tishkoff S.A., Singh L., Thangaraj K., Villems R., Comas D., Sukernik R., Metspalu M., Meyer M., Eichler E.E., Burger J., Slatkin M., Pääbo S., Kelso J., Reich D., Krause J. 2014, « Ancient human genomes suggest three ancestral populations for present-day Europeans ", dans Nature, $\mathrm{n}^{\circ} 513$ : 409-413.

Pääbo S. 1985, " Molecular cloning of Ancient Egyptian mummy DNA ", dans Nature, $n^{\circ} 314$ : 644-645.

Prüfer K., Racimo F., Patterson N., Jay F., Sankararaman S., Sawyer S., Heinze A., Renaud G., Sudmant P.H., de Filippo C., Li H., Mallick S., Dannemann M., Fu Q., Kircher M., Kuhlwilm M., Lachmann M., Meyer M., Ongyerth M., Siebauer M., Theunert C., Tandon A., Moorjani P., Pickrell J., Mullikin J.C., Vohr S.H., Green R.E., Hellmann I., Johnson P.L., Blanche H., Cann H., Kitzman J.O., Shendure J., Eichler E.E., Lein E.S., Bakken T.E., Golovanova L.V., Doronichev V.B., Shunkov M.V., Derevianko AP., Viola B., Slatkin M., Reich D., Kelso J., Pääbo S. 2014, « The complete genome sequence of a Neanderthal from the Altai Mountains ", dans Nature, ${ }^{\circ} 505$ : 43-49.

Rasmussen M., Li Y., Lindgreen S., Pedersen J.S., Albrechtsen A., Moltke I., Metspalu M., Metspalu E., Kivisild T., Gupta R., Bertalan M., Nielsen K., Gilbert M.T., Wang Y., Raghavan M., Campos P.F., Kamp H.M., Wilson A.S., Gledhill A., Tridico S., Bunce M., Lorenzen E.D., Binladen J., Guo X., Zhao J., Zhang X., Zhang H., Li Z., Chen M., Orlando L., Kristiansen K., Bak M., Tommerup N., Bendixen C., Pierre T.L., Grønnow B., Meldgaard M., Andreasen C., Fedorova S.A., Osipova L.P., Higham T.F., Ramsey C.B., Hansen T.V., Nielsen F.C., Crawford M.H., Brunak S., Sicheritz-Pontén T., Villems R., Nielsen R., Krogh A., Wang J., Willerslev E. 2010, "Ancient human genome sequence of an extinct PalaeoEskimo", dans Nature, n 463 : 757-762. 
Rasmussen M., Anzick S. L., Waters M. R., Skoglund P., DeGiorgio M., Stafford T. W. Jr., Rasmussen S., Moltke I., Albrechtsen A., Doyle S.M., Poznik G.D., Gudmundsdottir V., Yadav R., Malaspinas A.S., White S.S., Allentoft M.E., Cornejo O.E., Tambets K., Eriksson A., Heintzman P.D., Karmin M., Korneliussen T.S., Meltzer D.J., Pierre T.L., Stenderup J., Saag L., Warmuth V.M., Lopes M.C., Malhi R.S., Brunak S., SicheritzPonten T., Barnes I., Collins M., Orlando L., Balloux F., Manica A., Gupta R., Metspalu M., Bustamante C.D., Jakobsson M., Nielsen R., Willerslev E. 2014, "The genome of a Late Pleistocene human from a Clovis burial site in western Montana ", dans Nature, $\mathrm{n}^{\circ} 506$ : 225-229.

Reich D., Green R.E., Kircher M., Krause J., Patterson N., Durand E.Y., Viola B., Briggs A.W., Stenzel U., Johnson P.L., Maricic T., Good J.M., Marques-Bonet T., Alkan C., Fu Q., Mallick S., Li H., Meyer M., Eichler E.E., Stoneking M., Richards M., Talamo S., Shunkov M.V., Derevianko A.P., Hublin J.J., Kelso J., Slatkin M., Pääbo S. 2010,
"Genetic history of an archaic hominin group from Denisova Cave in Siberia », dans Nature, $\mathrm{n}^{\circ} 468$ : 1053-1060.

Schiffels S., Haak W., Paajanen P., Llamas B., Popescu E., Loe L., Clarke R., Lyons A., Mortimer R., Sayer D., Tyler-Smith C., Cooper A., Durbin R. 2016, » Iron Age and Anglo-Saxon genomes from East England reveal British migration history ", dans Nature Communications, ${ }^{\circ} 7: 10408$.

Skoglund P. \& Mathieson I. 2018, « Ancient Genomics of Modern Humans: The First Decade ", dans Annual Review of Genomics and Human Genetics, $\mathrm{n}^{\circ} 19$ : 381-404.

Slon V., Hopfe C., Wei C.L., Mafessoni F., de la Rasilla M., Lalueza-Fox C., Rosas A., Soressi M., Knul M.V., Miller R., Stewart J.R., Derevianko A.P., Jacobs Z., Li B., Roberts R.G., Shunkov M.V., de Lumley H., Perrenoud C., Gušić I., Kućan Ž., Rudan P., Aximu-Petri A., Essel E., Nagel S., Nickel B., Schmidt A., Prüfer K., Kelso J., Burbano H.A., Pääbo S., Meyer M. 2017, «Neandertal and Denisovan DNA from Pleistocene sediments ", dans Science, $n^{\circ} 356: 605-608$.

Caroline COSTEDOAT est Maître de conférences-HDR en génétique des populations à Aix- Marseille Université au sein de l'UMR ADES 7268 (AMU, CNRS, Établissement Français du Sang PACA Corse). Ces activités de recherches en anthropologie génétique se concentrent notamment sur l'étude des relations génome-environnement et sur des contextes d'identification populationnelle et individuelle du passé.

caroline.costedoat@univ-amu.fr

Stéphane MAZIERES est anthropologue généticien, Chargé de recherche au CNRS au laboratoire ADES, UMR7268 (Aix Marseille Université, CNRS, Etablissement Français du Sang PACA Corse). Ses travaux portent essentiellement sur l'étude de la diversité génétique humaine, la dynamique des peuplements, et l'étude de la répartition des polymorphismes du globule rouge entre les populations. stephane.mazieres@univ-amu.fr 


\section{Notes}

1 Discipline scientifique qui étudie les restes fossiles des êtres vivants du passé et les implications évolutives ressortant de l'étude de ces restes.

2 Étude de l'histoire évolutive des organismes vivants par l'établissement de leurs liens de parenté (phylogénie), et ce à partir de séquences de macromolécules biologiques tel que l'ADN.

3 Le principe du clonage moléculaire par transformation bactérienne consiste à multiplier un fragment d'ADN par l'intermédiaire d'un micro-organisme (souvent la bactérie E. coli).

4 Polymerase Chain Reaction (ou réaction de polymérisation en chaînes) est une méthode de biologie moléculaire d'amplification génique in vitro. Elle permet de dupliquer en grand nombre une séquence d'ADN ou d'ARN connue, à partir d'une faible quantité d'acide nucléique.

5 Les NGS ne fournissent pas la séquence complète d'un génome, mais seulement un très grand nombre de courtes séquences issues de ce même génome (appelés « reads »). On obtient un grand nombre de reads qui vont recouvrir plus ou moins les différentes bases du génome. Cette couverture génomique est symbolisée par $X$ et est généralement de l'ordre de quelques dizaines. Un séquençage à 10x contient donc, en moyenne, 10 fois chaque paire de bases du génome, plus le $X$ est grand, meilleure est la résolution du séquençage. Les reads obtenus se chevauchent partiellement et il faut donc ensuite reconstruire le puzzle à partir de ces chevauchements, pour obtenir le génome initial. 